\title{
Modelling purposeful processes based on the geometric representation of their trajectories
}

\author{
Igor Skopin ${ }^{1,2}$ \\ ${ }^{1}$ The Institute of Computational Mathematics and Mathematical Geophysics SB RAS \\ (ICM\&MG SB RAS) \\ 6, Ac. Lavrentieva ave., Novosibirsk, 630090, Russia \\ ${ }^{2}$ Novosibirsk State University (NSU) \\ 1, Pirogova st., Novosibirsk, 630090, Russia
}

\begin{abstract}
The possibilities of studying processes based on the geometric representation of their trajectories in a multidimensional space of factors and using projections of trajectories on planes suitable for analysis are discussed. A connection is established between such constructions and the spiral and snail of development. Based on the proposed projection, a development snail is defined as a special representation format of the purposeful process, expanding the possibilities of analysis of modelling results.
\end{abstract}

\section{Introduction}

Carrying out any research and, in particular, the study of developing processes is always based on a certain methodological base, which even when not explicitly formulated, largely determines the methods used and their instrumental support. The quality and effectiveness of the research work depend on how adequate this support is: the accuracy of forecasts, management efficiency, etc. The foregoing fully relates to the study of processes for which a criterion for the quality of development is defined as an a priori specified function on a variety of factors, the so-called purposeful processes. Such processes are often multifactorial and interdisciplinary, i.e. developing under the influence of multidirectional factors related to various aspects of reality.

Studies of multifactor purposefully developing processes are relevant for many applied areas, both technical and social. At the same time, when modelling such processes, interdisciplinary requires the coordination of models that are built for different aspects of the processes under study. This task needs special methods and tools with adequate support for comparing aspect calculations, agreed development options and, ultimately, determining optimal trajectories of the processes. The purpose of this article is to discuss the feasibility of developing such a tool, the conceptual basis of which is the geometric representation of possible trajectories as curves of a special kind in the space of factors.

The idea of the proposed approach goes back to the method of studying economic processes using their representation in the form of a triple helix twisted around the time axis, which Badulin develops in $[1,2]$. The projection of such a helix onto the plane of constant time, called the development snail, is divided into sectors, each of which is characterized by its own specific control. The method allows you to distinguish between factors that affect development in different phases of the process. This model 
structure makes it possible to analyze multiphase multifactor processes in various aspects, using criteria for assessing the quality of development common to all phases. When modelling economic processes, such criteria ultimately come down to cost characteristics and investment attractiveness of projects.

In [3], we examined the possibility of extending the idea of a snail to research in other areas when it is required to study the purposeful development of simulated processes. We propose the formalization of information handling, which was used by Badulin in his approach. This formalization describes data processing tools that are consistent with the well-established method of analysis using the so-called $S$ curves [4]. Based on this, we presented a sketch of the architecture of the instrumental system for supporting the study of processes for which development criteria are defined.

In this article, the proposed approach is expanded due to the possibility of projecting trajectories on different planes of various dimensions of the factor space. Accordingly, additional tools are introduced that are useful in multivariate analysis to coordinate autonomous aspect models of processes. Section 2 introduces the basic concepts used in the construction of projections of trajectories of the development of dynamic processes. It also defines the characteristic properties of correct trajectories - the proposed approach can be applied only when the development trajectories of the process satisfy the presented correctness condition, which is quite natural. Section 3 discusses various aspects of the concept of processes phases and transitions of phases as well as tools for development analysis and suggests approaches to phase decomposition of a process. Section 4 is devoted to formalizing the notion of admissibility of a trajectory and assessing the quality of a process using a geometric representation of development in the form of cones of all possible and permissible trajectories. The methodology for organizing factor analysis based on projection is given in section 5 . The next section 6 covers the concept of time, reflecting the needs of development modelling. On this basis, the boundaries of the adequate application of the developed approach are determined. Section 7 establishes the correspondence of the proposed approach and the methods of development analysis based on the cochlea. In conclusion, a sketch of the toolkit architecture is given, in which the requirements presented in [3] are supplemented by new means for model information operating.

In order not to limit the scope of any practical applications of our approach, the proposed materials are presented formally and are only accompanied by illustrative examples.

\section{Criterion and trajectory of a purposefully developing process}

The basic concept for the proposed approach is a development criterion, defined as a function (functional) $F$, depending on the time and combination of factors $a_{1}, \ldots, a_{n}$, which affect the process, and which the process affects, generating values from the set with a full order relation. For definiteness, real numbers are considered as the range of values of $F$.

Economic indicators can serve as good examples of the development criterion: income minus the costs of the project and the distribution of this value over the execution time of the simulated process, possibly with a discount. It is with such criteria Badulin works in $[1,2]$.

An example of a different kind is from the field of oilfield design, when it is necessary to estimate the oil recovery coefficient, i.e. ratio of recoverable oil to the value of geological reserves. This indicator of field operation accumulates during development modelling, integrally characterizing the chosen production strategy. The volumes of recoverable oil, distributed over time, depend on many factors associated with model variants of operating schemes.

A third example of a criterion can be used to reflect the stability index when it is important that the development path remains within predetermined limits. This case is typical in the simulation of device management, that optimizes the operating mode. An illustration of it is given in [2], where we consider a sketch of a model of the process of heating a steam boiler and discuss the criteria and requirements associated with this process.

Presented and some others criteria are formally equivalent - you can always choose a criterion of one type to simulate the functions of another. As always happens in such cases, this equivalence should not be misleading: in practice, the choice of a criterion and its type is one of the first tasks that must be solved when embarking on modelling development. Depending on the objectives of the study, the 
possibilities of obtaining reliable information about reality and other features, we can talk about the preference of a particular option. These circumstances should be considered in modelling support tools.

It is believed that for a fixed initial moment of nucleation of the process $t=0$ and its completion time $t=E$ any trajectory of development is a directional curve $\mathfrak{C}$ in $n+2$-dimensional space (time, development criterion $F$ plus $n$ factors $a_{1}(t), \ldots, a_{n}(t)$ that change over time) which points $R, R^{\prime}, R^{\prime \prime}$ and others are given by the formula:

$$
R=\left(t, F\left(t, a_{1}(t), \ldots, a_{n}(t)\right), a_{1}(t), \ldots, a_{n}(t)\right), 0 \leq t \leq E .
$$

The trajectory begins at a point on the plane $t=0$, called the initial one, which corresponds to the values of factors in the initial state of the simulated process:

and ends at the End point:

$$
\text { Begin }=\left(0, F\left(0, a_{1}(0), \ldots, a_{n}(0),\right), a_{1}(0), \ldots, a_{n}(0)\right),
$$

$$
\text { End }=\left(E, F\left(E, a_{1}(E), \ldots, a_{n}(E)\right), a_{1}(E), \ldots, a_{n}(E)\right) .
$$

The correct trajectory is represented by a curve for which the following conditions are true:

- $\forall R^{\prime}, R^{\prime \prime} \in \mathbb{C}\left(R^{\prime} \Varangle R^{\prime \prime}\right) \equiv\left(t\left(R^{\prime}\right)<t\left(R^{\prime \prime}\right)\right)$,

- $\forall \tau \in[0, E] \exists R(R \in \mathbb{C}) \&(t(R)=\tau)$,

- $\forall R^{\prime}, R^{\prime \prime} \in \mathbb{C}\left(R^{\prime} \neq R^{\prime \prime}\right) \supset \neg\left(t\left(R^{\prime}\right)=t\left(R^{\prime \prime}\right)\right)$;

where $\Varangle$ is an order relation on the set of points of the curve $\mathfrak{C}$ : the initial point is minimum the starting point is the minimum in the sense of this order, and the end point is maximum; $t(R), t\left(R^{\prime}\right)$ and $t\left(R^{\prime \prime}\right)$ are the values of the time coordinates of the points $R, R^{\prime}$ and $R^{\prime \prime}$ of curve $\mathfrak{C}$, accordingly.

These conditions are equivalent to the following statement:

The correct trajectory intersects with any plane of constant time $t=\tau \in[0, E]$ at exactly in one point; if the $\tau$ is outside of the specified interval, then the trajectory does not intersect with such a plane.

If $R^{\prime} \Varangle R^{\prime \prime}$ is valid for the points $R^{\prime}$ and $R^{\prime \prime}$ of the correct trajectory $\mathfrak{C}$, then we say that $R^{\prime}$ is located to the left of $R^{\prime \prime}$, and $R^{\prime \prime}$ is to the right of $R^{\prime}$. In accordance with the correctness conditions, this means the same as $t\left(R^{\prime}\right)<t\left(R^{\prime \prime}\right)$, i.e. temporal ordering of points: $R^{\prime}$ before $R^{\prime \prime}$ and $R^{\prime}$ later than $R^{\prime}$.

These limitations on curves correspond to realistically feasible trajectories and reflect the relationships and dependence of factors are additionally specified. They fix a set of curves containing all curves that meet the development criterion of the process. Information for a more precise definition of this set is extracted from a priori agreements, from laws characteristic of the applied field and for the problems being solved. A productive source of data on the relationships between factors in the dynamics of their development are autonomous process models that reflect different aspects of the main purposefully developing process. Such models are hereinafter referred to as aspectual. As the representation of the trajectories, only those curves from the $n+2$-dimensional space are used, the points of which satisfy the conditions for the correctness of the trajectory and the conditions associated with a priori agreements and with the relations obtained in aspect modelling.

\section{Phases and phase transitions of a purposefully developing processes}

The implementation of real processes is often convenient to consider as a sequence of stages, or phases, which are characterized by developmental changes that change over time. For controlled processes, for which external influences lead to the process goal, the phases change each other due to a directed change in development factors. Phase transitions of physical, chemical and other processes of the real world, the goals of which are attributed by the researcher, are subject to the laws of nature. At the formal level of consideration, there are no other grounds for distinguishing phases and transitions between them, except for the features of the trajectory as a curve in the space of factors: points of the beginning, end of the curve, its extrema and inflections. In accordance with the correctness condition for the trajectory for each of these points, it is possible to construct parallel planes of constant time. Parts of the trajectory between these adjacent planes are considered as a formal geometric representation of the phases of the process, and the planes themselves as a geometric representation of phase transitions.

The formal presentation of the phases of the process has only an indirect relation to the meaningful interpretation - it is hard to expect that it will always be possible to interpret such phases as independent 
elements of the model. For real processes, instead of formal phases, it may be useful to divide the curve of the trajectory into sections in which a set of factors is known that most significantly affect the process, and then it is natural to associate the transition from one phase to another with a change in significant factors.

If the developer of the model knows well the scope of the problem being solved, then it is not difficult for him to indicate factors that are essential for well-defined periods of development. An illustration of this is the already mentioned study by Badulin [1,2], which builds a model of the life cycle of an innovative business, based on the allocation of four types of resources: people, technology, capital and the market, considered as the main factors of development. Each of them goes through three stages of change. Thus, twelve phases of the studied process appear, characterized by their essential factors and their control. A single development criterion for the entire process, reflecting the economic assessment of the process, should be adjusted at each phase: in the early phases, the management task is reduced to the effectiveness of analyzing the primary idea and bringing it to a market product, which will give an economic effect only in the later phases involving sales.

Figure 1 shows a slightly modified scheme for developing an innovative business from [2] in the form of a snail unwinding from the first phase transition. The process begins from in the point $\boldsymbol{T}$ The process begins (marked with a circle and signature) and develops a clockwise direction. The phases are depicted as snail sectors. The dashed lines indicate the transition of the business to the next stage in two possible variants: successful development and degradation of the business.

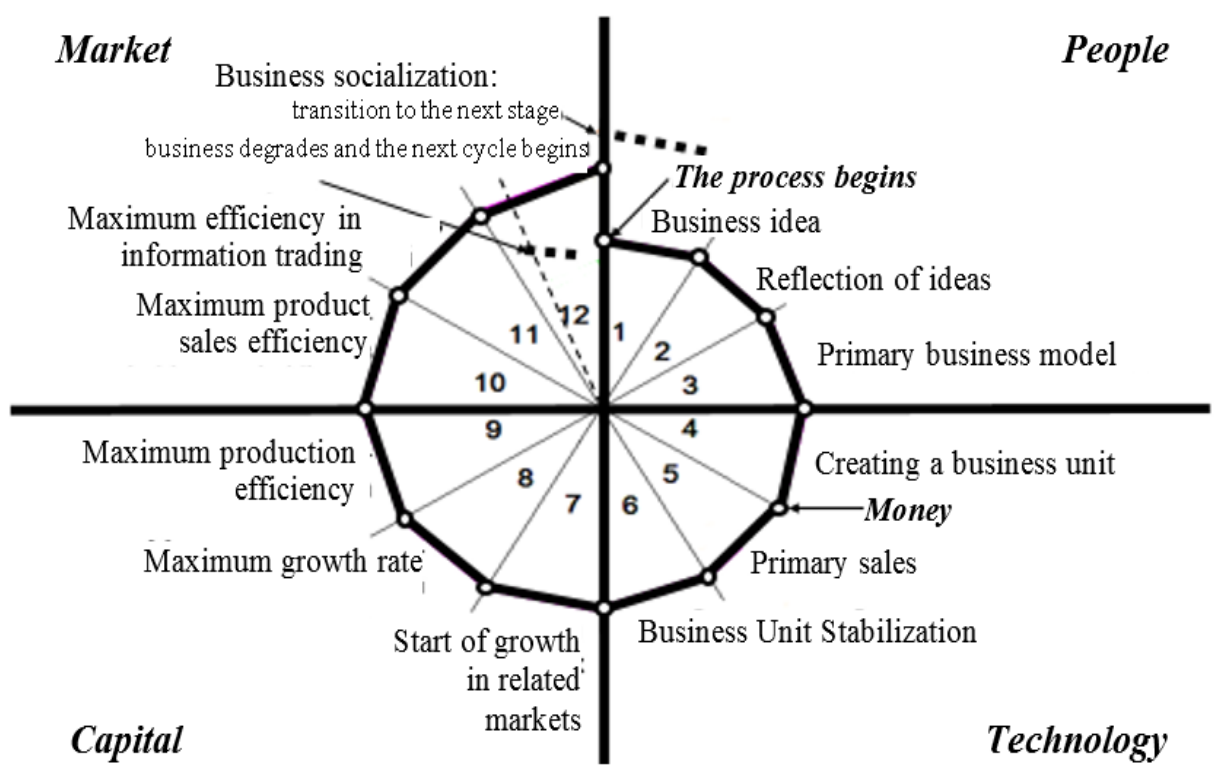

Figure 1. Snail development of innovative business.

The diagram shows that only after the phase transition of Money to the fifth phase of the process do economic factors appear that significantly affect the process. In addition, the scheme demonstrates a characteristic feature of the phase decomposition of processes based on the significance of factors: the ambiguity of phase separation. The allocation of four types of resources, shown as quadrants of a plane, is nothing more than an alternative decomposition option. It can be considered, and for some studies it is advisable, such enlarged phases as independent processes. If the similar decomposition is carried out systematically, then in the cases under consideration a hierarchical system of processes is built. In this cases, the goal of modelling at the lower levels is to supply information to processes at the higher hierarchical levels. 
Another approach to the phase decomposition of a developing process is demonstrated by the task of determining the life of an incandescent lamp from [3]. Its deciding model is built as a series of elementary acts, each of which consists of switching on, burning, and switching off. Performing a sequence of elementary acts leads to the accumulation of fatigue, and at the end of the process to burnout of the lamp. The trajectory of each of the acts is determined by factors due to physical laws that connect the current strength, voltage, resistance and temperature of the spiral, as well as reflecting the gradual fatigue (evaporation) of the spiral. In accordance with them, a trajectory curve is built in the space of factors with many special points determined by physical laws and, accordingly, formal phases. But most of them are redundant to determine the operating life - it is enough to take into account the dynamics of only those factors, due to which fatigue is growing. Therefore, it is advisable to determine the meaningful phases of the operation process as repeatable parts of the trajectory, each of which corresponds to one elementary act. So certain phases combine the formal phases associated with switching on, burning, and switching off. If the factors acting on the switched off lamp are essential for determining accumulated fatigue, then the phases of elementary events must be alternated with additional phases that take into account the cooling process of the spiral. In this case, the separation of the essential factors of these two types of phases is quite obvious, and the decomposition of the model, which was mentioned above, is real: the phases of elementary acts correspond to aspect modelling, which provides the information necessary for the basic process to determine the service life.

Figure 2 illustrates a phase decomposition with repeating phases for an incandescent lamp problem. As in the previous case, two phase levels can be seen in this decomposition. At the upper level, a fatigue curve is formed from fragments that line up as repeating phases of modelling elementary acts.

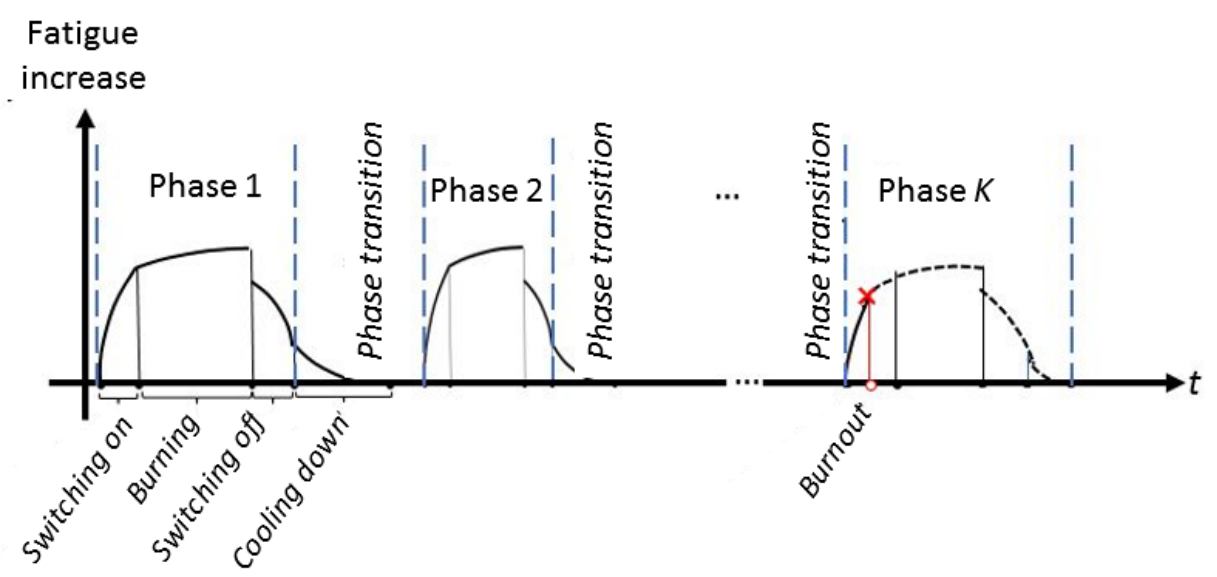

Figure 2. Phase decomposition in the task of determining the lamp life.

The presented illustrations reflect two opposite possibilities of phase structures of processes. More complex processes may require a combination of aspect models with non-repeating and repeatable phases. It is necessary that these and other similar cases of real model construction be described as adequately as possible to reality. In particular, the concept of factors' essentiality affecting the purposeful processes must be precisely defined. In the framework of the geometric formalism developed in this paper, this concept is specified by a series of the following definitions:

a) Let $R$ be a curve point $\mathbb{C}$ and $\tau=t(R)$ is its time coordinate. The development criterion of the process at this point is $F\left(\tau, a_{1}(\tau), \ldots, a_{n}(\tau)\right)$. Let further $R^{\prime}$ is non-curve $\mathfrak{C}$ point which differs from $R$ only in the value of the factor $a_{i}, i \in\{1, \ldots, n\}$, and its new value $a_{i}^{\prime}$ is valid for this factor. Then, if among all such points there is at least one for which

$$
\left|F\left(\tau, a_{1}, \ldots, a_{i}(\tau), \ldots, a_{n}\right)-F\left(\tau, a_{1} \ldots, a_{i}^{\prime}(\tau), \ldots, a_{n}\right)\right|>\Delta,
$$

where $\Delta$ is a priori given threshold of distinguishability of criterion values, then factor $a_{i}$ it is considered significant at the point $R$, which is denoted as $a_{i} \bowtie R$. 
b) The set of factors $M \subset\left\{a_{1}, \ldots, a_{n}\right\}$ is called essential at the point $R \in \mathfrak{C}$, if $\forall a \in M(a \backsim R)$, which is denoted by M $\leadsto$ R.

Further notions are defined for open intervals of trajectories:

c) If $R^{\prime}, R^{\prime \prime} \in \mathfrak{C}$ are points of the correct trajectory $\mathfrak{C}$, for which $R^{\prime} \Varangle R^{\prime \prime}$, then part of the curve $\mathfrak{C}$ between these points, denoted by $I=\left(R^{\prime}, R^{\prime \prime}\right)$, is called as open interval of trajectory, and It $=\left(t\left(R^{\prime}\right), t\left(R^{\prime \prime}\right)\right)$ is open time interval generated by $I$.

The correct path conditions guarantee that $I \neq \emptyset$, as well as the uniqueness of the correspondence between $I$ and $I t$.

- A factor $a_{\mathrm{i}}$ from the set $\left\{a_{1}, \ldots, a_{n}\right\}$ is called essential on the interval $I=\left(R^{\prime}, R^{\prime \prime}\right)$ of the curve $\mathbb{C}$, if $\forall R \in I\left(a_{i} \bowtie R\right)$, which is denoted as $a_{i} \bowtie I$.

- The set of factors $M \subset\left\{a_{1}, \ldots, a_{n}\right\}$ is called essential on the interval $I=\left(R^{\prime}, R^{\prime \prime}\right)$ of the curve $\mathbb{C}$, if $\forall R \in I \forall a \in M(a \leadsto R)$, which is denoted as $M \bowtie I$.

For two intervals $I^{1}=\left(R_{1}^{\prime}, R_{1}^{\prime \prime}\right)$ and $I^{2}=\left(R_{1}^{\prime}, R_{1}^{\prime \prime}\right)$ of the curve of the trajectory $\mathfrak{c}$ such that $I^{2} \subset I^{1}$, is correct the statement:

If $M \subset\left\{a_{1}, \ldots, a_{n}\right\}$ is the set of essential factors on $I^{1}$, then $M$ is the set of essential factors on $I^{2}$.

This obvious property makes it meaningful to find the widest possible intervals by their duration. For a given $M$, it is required to find the maximum interval $I=\left(R^{\prime}, R^{\prime \prime}\right)$, i.e. such that at a fixed left or right boundary the maximum $t\left(R^{\prime \prime}\right)-t\left(R^{\prime}\right)$ is reached. Its solution is used to set the phase decomposition of the process on the basis of the relation of materiality: the concept of phases is associated with intervals of the trajectory with the most significant factors for them.

The construction of decomposition begins with specifying a collection of sets of materiality of factors $\mathfrak{M}=<M^{1}, \ldots, M^{K}>, M^{k} \subset\left\{a_{1}, \ldots, a_{n}\right\}, 1 \leq k \leq K$. At the formal level of consideration, no restrictions are imposed on sets from $\mathfrak{M}$, in particular, the repetition of $M^{k}$ is allowed, which is interpreted as the possibility of re-performing phases during the development of the process. Distributing the factors according to $M^{k}$ and ordering $\mathfrak{M}$ in accordance with the necessary sequence of phases, the model designer is guided by informative information about the simulated reality - this is the basis of the phase decomposition of the process and, in particular, the choice of strategy for its construction.

Next, we will present two fundamentally different strategic schemes that can be considered as a reconstruction of how the two illustrations presented above could appear: the model of the life cycle of an innovative business and the task of determining the life of an incandescent lamp.

- For the first scheme, it is postulated that $M^{k}$ in $\mathfrak{M}$ are presented once, which corresponds to the idea of the uniqueness of the phases of the process associated with materiality factors from $M^{k}$.

- For the second scheme, the opposite strategy is chosen: the phases associated with the combustion factors of the lamp are repeated until fatigue reaches its limit value. In order not to overload the presentation with the details of real decomposition, specific factors associated with the discussed schemes are not considered - this is the task of constructing a real decomposition of the processes.

The decomposition of a developing process with non-repeating phases is presented in the form of the following algorithm.

Step 0. Initialization.

Set the number of the first set of essentialities $k:=1$, as well as the working part of the trajectory $\mathbb{C}^{k}:=\mathfrak{C}$, which will be used to construct the decomposition in step 1.

Define the set of maximal intervals $\Omega$ in $\mathbb{C}^{k}$, with the essentiality set $M^{k}$ :

$$
\Omega=\left\{I=\left(R^{\prime}, R^{\prime \prime}\right) \&\left(M^{k} \bowtie I\right)\right\} \text {, i.e. such that } \forall I, I I \in \Omega((I \neq I I) \supset(I \cap I I=\emptyset)) .
$$


From the set $\Omega$, choose the interval $I$ with the smallest left boundary $R^{\prime}$. As a result, the curve $\mathbb{C}^{k}$ is divided into the intervals $I^{L}, I$, and $I^{R}: \mathfrak{C}^{k}=\bar{I}^{L} * I * \overline{I^{R}}$, where* is the concatenation operation, and $\overline{I^{L}}$ and $\overline{I^{R}}$ are the closure of the open intervals $I^{L}$ and $I^{R}$ by joining the boundary points to them.

End Step 0.

Step 1. Beginning of the construction of the phase number $k$ - determination of its left boundary.

$I^{L}$ may turn out to be a degenerate empty interval containing no points, i.e. $I^{L}=\left(R^{L^{\prime}}, R^{L^{\prime \prime}}\right)$, where $R^{L \prime}=R^{L \prime \prime}$. In this case, the boundary point $I^{L}$ is considered as a phase transition to the phase with number $k$, the construction of the left boundary of which is completed.

If $I^{L}$ contains other points, then this entire interval becomes the phase transition to the phase with number k. Attach $I^{L}$ to $I$, i.e. $I:=I^{L} * R^{L^{\prime \prime}} * I$, or not, remains at the discretion of the developer.

End Step 1.

Step 2. Continuation of the construction of the phase number $k$.

Remove the interval $I$ from $\Omega$, i.e. $\Omega:=\Omega \backslash I$. If the new set $\Omega=\emptyset$, then this step is completed. Otherwise, the developer has two possibilities:

a) Select the interval $I I=\left(R^{I I^{\prime}}, R^{I I \prime \prime}\right)$ in $\Omega$ that has the smallest left boundary $R^{I I \prime}$ and to use it for the extension $I$ on the right: $I:=I *\left[R^{\prime \prime}, R^{I I \prime}\right] * I I$, where $\left[R^{\prime \prime}, R^{I I \prime}\right]$ is a closed, possibly degenerate $\left(R^{\prime \prime}=R^{I \prime \prime}\right)$ interval that separates $I$ and $I I$. The points of this interval are declared as special for the phase under construction.

Correct the partition of the working part of the trajectory $\mathbb{C}^{k}:=\bar{I}^{L} * I * \overline{I^{R}}$.

Repeat Step 2 until $\Omega$ is exhausted, or option (b) is selected.

b) Ignore the presence in $\Omega$ of intervals that could be used for extension $I$. As a result, the partition of the curve $\mathfrak{C}^{k}=\bar{I}^{L} * I * \bar{I}^{R}$ does not change.

End Step 2.

Step 3. Completion of the construction of the phase number $k$.

a) If $k=K$ then the last phase is constructed, and $I^{R}$ is phase transition completing the simulated process. The agreement on whether it joins the phase number $k$ by interval $I=\left(R^{\prime}, R^{\prime \prime}\right)$ or not remains at the discretion of the developer.

b) If $k<K$ then to construct the phase using the set $M^{k+1}$, it is necessary to form a new working part of the trajectory $\mathbb{C}^{k+1}$. It is nothing but the definition of its left boundary. Depending on the features of the simulated process, the developer determines whether the intersection or even the embedding of the phases into each other is permissible ${ }^{1}$. In this case, one of the following conditions must be met:

- If phase intersection is not allowed, then inside the interval $I^{R}$ there must exist at least one interval $I I$ such that $M^{k+1} \bowtie I I$

- If phase intersection is allowed, then the existence of the interval $I I=\left(R^{I I^{\prime \prime}}, R^{I I \prime \prime}\right)$ for which $M^{k+1} \backsim I I$ is supplemented by the requirement that this interval be part of $I * \overline{I^{R}}$.

Thus, the working part of the trajectory $\mathfrak{C}^{k+1}$ for constructing the phase number $k+1$ is defined as $\overline{I^{R}}$, possibly extended on the left by that part of the interval $I$ for which phase intersection is allowed. For $\mathfrak{C}^{k+1}$ the set of maximum intervals $\Omega=\left\{I=\left(R^{\prime}, R^{\prime \prime}\right) \&\left(M^{k+1} \bowtie I\right)\right\}$ is updated: a new interval $I$ with

\footnotetext{
${ }^{1}$ Use overlapping phases in modelling when the developing process is necessary to reflect the joint or parallel execution of related phases.
} 
the smallest boundary $R^{\prime}$ is deleted from $\Omega$. Accordingly, the partition $\mathfrak{\complement}^{k+1}$ is renewed: $\mathfrak{\complement}^{k+1}=\bar{I}^{L} *$ $I * \overline{I^{R}}$.

The last steps in Step 3 are to assign $k:=k+1$ and go to Step 1 .

End Step 3.

The presented trajectory decomposition algorithm allows one to check the correctness of the specification of the sets $M^{k}$ and, as the construction progresses, make the necessary corrections to the decisions made. It is connected with the innovation business development scheme only in that phase repetition is not allowed and phase intersection is allowed. This method is applicable in all cases when these conditions correspond to the specifics of a particular simulation.

In this sense, the following illustration is the opposite: in the method for solving the problem of determining the life of an incandescent lamp, it was decided that the experiment is built as a series of repeating alternating elementary acts of combustion and cooling. In the model, on any correct trajectory representing the process, the intervals of the curve corresponding to the burning times are separated by cooling intervals (possibly degenerate if simultaneous switching off and on of the lamp is allowed). In essence, this means that the process is divided into phases of the same type and phase transitions from one to another are determined. In addition to such transitions, the initial and final phase transitions are especially distinguished. The first of them, or rather the event associated with it, is special, because it sets the initial settings of the factor values for the entire process, and the second - because it stops the process.

Formally, a fairly large number of factors that could be considered significant are associated with the so-defined phases: voltage, current, temperature of the filament, etc. But to construct a decomposition, it is enough to select only one of them, the significance of which determines all the others. Substantially, this is the closure of the circuit containing the lamp, the amperage current strength can serve as a numerical characteristic of it. It is positive when the lamp is on, and is zero otherwise. This convention leads to the fact that for any trajectory the collection of essential sets of factors $\mathfrak{M}=\left\langle M^{1}, \ldots, M^{K}\right\rangle$, $1 \leq k \leq K$, where all $M^{k}$ are equal to the singleton set $\{$ Amperage $\}$. The number $\mathrm{K}$ is determined during the execution of model calculations: lamp burnout is a signal to stop the simulation. As a criterion for the development of the process, it is natural to choose a fatigue factor, the accumulation of which over a certain limit leads to burnout.

For further construction, it is necessary to clarify the notion of essentiality, because according to the previous definition, the Amperage factor does not differentiate the intervals: for $a_{i}=$ Amperage, the relation

$$
\left|F\left(\tau, a_{1}, \ldots, a_{i}(\tau), \ldots, a_{n}\right)-F\left(\tau, a_{1} \ldots, a_{i}^{\prime}(\tau), \ldots, a_{n}\right)\right|>\Delta
$$

holds for all points of the trajectory $\mathfrak{c}$ except the last one, which corresponds to burnout. Here a more general definition is needed, which is applicable in such cases. Instead of the specified ratio, justice is required

$$
\left|F\left(\tau, \ldots, \varphi^{a_{i}}\left(a_{1}, \ldots, a_{i}(\tau), \ldots, a_{n}\right), \ldots a_{n}\right)-F\left(\tau, \ldots, \varphi^{a_{i}}\left(a_{1}, \ldots, a_{i}^{\prime}(\tau), \ldots, a_{n}\right), \ldots, a_{n}\right)\right|>\Delta,
$$

where $\varphi^{a_{i}}$ is a certain a priori defined verification function that relates the values of the factors at the points $R$ and $R^{\prime}$ of the curve $\mathfrak{C}$, differing only in the value of the $i$-th factor.

In the case of modelling an incandescent lamp, it is quite reasonable to assume the presence of a factor indicator LampIsOn with values of 1 for the on lamp and 0 for the off. So LampIsOn*Amperage can be taken as $\varphi^{\text {Amperage }}$, which is zero when the lamp is off.

Following the new definition, the set of maximal intervals $\Omega$ in $\mathfrak{C}$ with the essentiality set $M^{k}=$ $M^{\text {Amperage }}$ is defined exactly as in the previous case: $\Omega=\left\{I=\left(R^{\prime}, R^{\prime \prime}\right) \&\left(M^{k} I\right)\right\}$ and with the same condition for maximizing its elements: $\forall I, I I \in \Omega \supset((I=I I) \vee(I \cap I I=\varnothing))$. The only difference is that the resulting sequence of intervals $I^{1}, \ldots, I^{K}, I^{k}=\left(R^{k \prime}, R^{k \prime \prime}\right), 1 \leq k \leq K$, gives a single phase decomposition

$$
\mathfrak{C}=R^{1 \prime} * I^{1} *\left[R^{1 \prime \prime}, R^{2 \prime}\right] * I^{2} * \ldots * I^{K} * R^{K \prime \prime},
$$

where $\left[R^{k \prime \prime}, R^{k+1 \prime}\right], 1 \leq k<K$, - closed cooling intervals (degenerate or non-degenerate). 
The presented phase decomposition strategies are just two examples illustrating the proposed approach. As was shown, depending on the needs of real modelling, the agreements that the developer follows when building, as well as the concepts associated with them, allow a certain freedom of choice. The determining arguments in the implementation of ur approach are related to the substantive features of the simulated process. The formal geometric representation of the phases and the relation of essentiality serve as nothing more than guidelines for the decomposition of the model.

The phases highlighted during the decomposition of the process can be considered independent processes performed in the corresponding time intervals with the development criterion inherited from the process forming them. This allows you to build a model decomposition along a hierarchical vertical: from the parent process model to the child ones embedded in it. For a nested model, many inherited factors can expand. This means the abstraction of the parent process from the added factors localized in the heir, the model of which is inherently aspectual. An illustration of the use of such abstraction is the second phase decomposition scheme, in which all sets of essentiality are presented as the single element: $M^{k}=M^{\text {Amperage }}$.

When constructing a system of models from parent and child models (which can also be decomposed), the set of such aspect models can be expanded by other processes, but this is already determined by the features of the application area.

Since the phase decomposition of simulated developing processes is often used in research, support for the means of operating with phases, phase transitions, as well as the determination of significant factors influencing the development of processes should be provided in the toolkit. Some more details about this theme one can found in [5].

\section{Admissibility of trajectories and assessment of the quality of the development of the process}

The following concepts are introduced so that when modelling, the developer has the opportunity to consider different options for the trajectories of the process, in particular, to compare them and choose the most appropriate to the problem being solved.

The construction of a process model begins with an indication of the conditions in which it is activated. This means setting the initial values of the factors, i.e. Begin point definition. Its fixation indicates all processes which trajectories begin in it. In the general case, the choice of the starting point is ambiguous, and it is assigned from some set DBegin lying in the plane $t=0$. This set is called the region of admissibility of the beginning of the trajectory. Thus, it is assumed the possibility of operating with different trajectories, for example, to compare the values of the criterion or other characteristics of the process. For the same reason, the time instant $T>0$ is determined for the end of trajectory tracking, at which the trajectory reaches the plane $t=T$ :

$$
\operatorname{End}(T)=\left(T, F\left(T, a_{1}(T), \ldots, a_{n}(T)\right), a_{1}(T), \ldots, a_{n}(T)\right) .
$$

The condition $T>0$ means that we exclude "instantaneous" processes from consideration, or, what is also, we associate purposeful development with the time periods during which the factors change.

For the specific trajectory of a process there are three following variants of ending:

- on the plane $t=T$, i.e. when $t=E$, and so $E n d(T)=E n d$;

- $\quad$ in front of the plane $t=T$, i.e. later $E$, and so $E n d(T) \Varangle E n d$;

- behind the plane $t=T$, i.e. earlier $E$, and so $E n d \Varangle E n d(T)$.

The last relation becomes correct if we formally continue the trajectory beyond the plane $t=T$ of a suitable curve ending at the point $E n d^{\prime}$ of this plane: $E n d(T)=E n d^{\prime}$.

Three options for ending the process are reducible to each other (see [3]), and therefore, to avoid excessive detail, we restrict ourselves to the case when the tracking end times and trajectories coincide. Accordingly, along with the set DBegin, two regions are defined: $D E n d$, which contains the intersections of all admissible trajectories with the plane $t=T$, and DResult, which contains the end of all admissible trajectories. The latter is called the results area of admissible trajectories. In further consideration, it is assumed that DResult $=$ DEnd. 
When modelling the development of the process, it is advisable to distinguish on the plane $t=T$ is the point of the most preferred completion of the process. For such a point, the vector (Begin, End) is considered as a special ideal trajectory that leads from a given starting point to a selectable final one. Further this vector is called the trend of development paths. The concept of a trend reflects the need for a model developer to have guidelines for comparing trajectories, and therefore should not be confused with any strategy that should always take into account the possibility of transition from one state to another, restrictions on permissible effects on the process, and other agreements, guaranteeing realism of modelling, even if idealized.

Evaluation of the quality of the development of the process can be carried out on the basis of calculating the function-criterion $F$ using one of three methods:

(1) final assessment $-F$ is calculated at the end of the tracking;

(2) extended estimation $-F$ is calculated at some moments $\tau_{i}$, i.e. at the selected points of the trajectory $R_{\tau_{i}}, 1 \leq i \leq k$, where $k$ is the number of such points;

(3) continuous evaluation $-F$ is calculated on demand, i.e. at any point on the trajectory when it is needed for analysis.

Final, extended and continuous evaluations are reducible to each other (see [3]). So taking in account on this, in the future we mainly talk about the final evaluations. It is clear that for real modelling the choice of another case means the need for appropriate adjustment of the constructions presented below, however, not very significant.

The central objects of a formalized representation of developing processes, as in the previous work [3], consider cones in the space of factors whose axes contain a trend vector (Begin, End):

1. The cone of all admissible trajectories that starting at the point Begin, which is considered as the vertex of the cone. Its axis coincides with the trend vector (Begin, End), and the base is the DResult results area of such trajectories.

2. The cone of all admissible trajectories is the union of cones over all points belonging to the DBegin region from previous item. This is a truncated cone with the bases DBegin and Result ${ }^{2}$, lying on the planes $t=0$ and $\mathrm{t}=\mathrm{T}$. The cone axis coincides with the trend vector for an Begin point selected on the basis of DBegin and calculated on the other basis of the End point.

3. The cone of the prehistory of the process under study. Its base is the DBegin region, the axis is the continuation of the trend vector in the direction of the negative time coordinate, at which a suitable vertex is selected. This is the cut-off part of the cone from item 2.

The consideration of the cone of prehistory is useful for taking into account the possibility of variants of the beginning of a trend. Whether or not a real prehistory exists, does not matter. Formally, this cone can be considered as a process that prepares the initial state for the process under study, if required in the study. If, as such process, the distinguished phase of another parent process is considered, then the prehistory is the phase preceding it, which is quite natural for such systems of process.

The continuation of the trajectories beyond the limits of the plane $t=T$ under no circumstances can affect their progress to the region of admissibility. Not every development leads to the fact that the End point is reached: it is possible that the process does not lead to the results area, which means that its trajectory is inadmissible. It makes sense to consider them, in particular, in order to find out the degree

\footnotetext{
${ }^{2}$ It would be more accurate to speak of minimal connected domains containing all points from DBegin and Result, respectively. Minimality can be understood as the choice of the region with the shortest border, with the smallest area or in any other sense, which corresponds to a clear desire to exclude from the consideration as much as possible insignificant.
} 
of approach of the trajectories to the target. As of course an impossible example of this kind, let us consider the modelling of oil production in the field, that illustrates unacceptable trajectories. In principle, a technology for enhancing oil recovery using a local atomic explosion is possible, which, of course, is not implemented due to environmental considerations, but at a model level it is possible to compare recommended for implementation cases with this unrealistic development option.

It is not possible to specify the exact form of the bases of the cones and their surfaces, since they substantially depend on the application area for which the model is being developed, on information provision, and on other aspects of model research. But this is not necessary - for analysis it is enough to know the ratios and intervals of the values of factors $a_{l}, \ldots, a_{n}$, which affect the development of the process. The same can be said about the permissible trajectories. For analysis, it is required to know not their specific form, but only periods of stationary behavior and moments of changing trends, i.e. transition from one stationary period to another. ${ }^{3}$ This can be done using, for example, two types of projection of the trajectories, discussed below.

\section{Projections of the development trajectory on the planes of constant time subspaces}

In [3] we show the productivity of using projections of the cones trajectories onto the plane (time, criterion) for analyzing developing processes, as well as the applicability limits of this method: it is able to give only an integrated assessment of processes, but does not show how individual factors influence on development. To overcome this drawback, this section proposes the method of constructing projections of trajectories using planes selected for various factors and their groups.

The planes onto which the trajectory is projected are set for each factor from $\left\{a_{1}, \ldots, a_{n}\right\}$ and some of their combinations by choosing a time coordinate equal to a constant. Which constant needs to use does not matter - for a fixed combination of factors, they will obviously give the same picture for any constant: each of the projection planes under consideration can be transferred to any other by suitable parallel translation. In particular, the equalities $t=0$ and $t=T$ lead to the same projection onto one of the bases of the cone of all trajectories. For definiteness, the first of these equalities is further adopted. Thus, projection planes $\left(F, C\left(a_{1}, \ldots, a_{n}\right)\right)$ are considered, where $\mathrm{C}$ is a certain vector function depending on factors (in the simplest case, their linear combination). The first coordinate of such planes is criterion $F$.

It should be noted that the vector $C$ inherits the dependence of factors on time, and therefore its choice is ambiguous, and it should be mediated by the decision about which estimation of the quality of the development of the process is taken for a specific study. In the final estimation strategy, the values of the factors should be selected at the end of tracking $T$, in case of an expanded one, it is necessary to specify projections for all moments of the phase transitions $\tau_{i}$, continuous evaluation requires the possibility of constructing projections at any time $\tau$ from the interval $[0, T]$. As mentioned above all types of assessment are reducible to each other, and therefore, remaining within the framework of a formalized abstraction, we discuss only the final assessment as the simplest. Among other things, this makes it easier to notate by omitting the time argument of factors, which is always equal to $T$.

Subsequently, the projection operator on $\left(F, C\left(a_{1}, \ldots, a_{n}\right)\right)$ is denoted as $\boldsymbol{P}_{\mathrm{C}\left(a_{1}, \ldots, a_{n}\right)}$ or as $\boldsymbol{P}$ if the identification $\mathrm{C}\left(a_{1}, \ldots, a_{n}\right)$ is implied. So, projection onto the plane $\left(F, a_{i}\right)$ is denoted by $\boldsymbol{P}_{a_{i}}$ or $\boldsymbol{P}$.

The problem of studying the projections $\boldsymbol{P}_{\mathrm{C}\left(a_{1}, \ldots, a_{n}\right)}$ consists in choosing the vector-function $\mathrm{C}$, which should reflect the combined contribution of factors to the success of the development of the process. Its solution is associated with the consideration of special functions, which normalized reflect the proportions of the influence of each factor on the development of the process depending on time and, thus, allow us to determine their correlation with the function-criterion $F$.

Further, these functions are denoted as $f_{i}(t), 1 \leq i \leq n$. The condition for their normalization is given by the ratio:

\footnotetext{
${ }^{3}$ If the trajectory could be considered a sufficiently smooth function, then stationarity would mean the constancy of all partial derivatives, and transitions between periods of stationarity — violations of this property.
} 


$$
\forall i\left(\left|f_{i}(t)\right| \in[0,1]\right) \& \sum_{i} f_{i}(t)=1 .
$$

When this relation is fulfilled, we can assume to a first approximation that

$$
C\left(a_{1}, \ldots, a_{n}\right)=\sum_{i} f_{i}(t) *\left(0, \ldots, 0, a_{i}, 0, \ldots, 0\right)
$$

reflects the combined influence of factors on $F$ at each point in time tracking. This vector-function is used hereinafter as the second coordinate of the main projection plane $\left(F, C\left(a_{1}, \ldots, a_{n}\right)\right)$. At the same time, it is useful to consider other projection planes, in particular, those defined by vectors

$$
f_{i}(t) *\left(0, \ldots, 0, a_{i}, 0, \ldots, 0\right) \text {. }
$$

They can provide information on the specific contribution of the $i$-th factor to $F$ (the multiplier $f_{i}(t)$ is used for comparability).

At each moment $t$, the functions $f_{i}(t)$ reflect the characteristic of the process associated with the use of some resource that has a certain valuation for the process: if the resource is spent, then $f_{i}(t)<0$, and if $f_{i}(t)>0$, it is produced. $f_{i}(t)=0$ indicates that at time $t$ the process does not produce and does not consume a resource. In particular, one can use $f_{i}(t)$ with totally zero values to denote case, when it is possible to use the resource without restrictions, and therefore it has no price for the process. This directly follows from the definition of $f_{i}(t)$. But it does not mean that $a_{i}$ is not a factor influencing the process - without its "infinite" presence, the process may not develop or even arise.

As an illustration of the "infinite" resource, let us consider one of the aspects of reality that can occur if it is necessary to simulate the development process of a company renting a room for work on a fixed fee. If the lease stipulates that the company does not bear the costs of maintaining the premises and, in particular, for the use of electricity, then the consumed electricity can be considered as an unlimited resource.

In cases where the resource $a_{i}$ is used without restrictions, it is advisable not to exclude it from the set $\left\{a_{1}, \ldots, a_{\mathrm{n}}\right\}$, but to artificially set the value $f_{i}(t)$ below the threshold value level during periods when necessary to take into account the presence of $a_{i}$.

If $\exists t_{1}, t_{2}\left(f_{i}\left(t_{1}\right)<0 \& f_{i}\left(t_{2}\right)>0\right)$, then the process consumes and produces the $i$-th resource.

The whole set of functions $f_{i}(t)$ forms the spectrum of $F$ and naturally splits the graph of this function into sections in which the influence of certain factors is stronger than the others. This is clarified by constructing the projections $\boldsymbol{P}_{a_{i}}$ for each $i \in\{1, \ldots, n\}$, which we discuss below. The information obtained can be used, for example, to correct the phase structure of the process, which may change when using the ratio of the significance of factors (see section 3). Thus, the model developer has additional tools for analysing the process, associated with S-curves or with other similar mappings of trajectories in the projection onto the plane (time, criterion) considered in [3]. The number of sites of the greatest influence of each of the factors and their size (duration) depend on the characteristics of the simulated processes.

The basis of the analysis of projections of the trajectory onto $\left(F, f_{i}(t) *\left(0, \ldots, 0, a_{i}, 0, \ldots, 0\right)\right)$ is the projection of the trend (Begin, End) onto this plane. If the development axis is parallel to the time axis, then the projection $\boldsymbol{P}_{a_{i}}$ (Begin, End) degenerates, i.e. turns into one point. Otherwise, projecting a segment (Begin, End) leads to a segment parallel to $\left(0, \ldots, 0, a_{i}, 0, \ldots, 0\right)$. This geometric characteristic reflects the tendency of the process with respect to individual factors. For definiteness, the direction of this vector is considered corresponding to the direction $\boldsymbol{P}_{a_{i}}($ Begin, End $)$.

When projecting related to factor $a_{i}$, the following situations are possible:

1. Over the entire trajectory, the resource is produced.

$$
\begin{aligned}
& \forall t\left(f_{i}(t) \geq 0\right) \\
& \forall t, t^{\prime}\left(\boldsymbol{P}_{a_{i}}(F(t)) \geq \boldsymbol{P}_{a_{i}}(\text { Begin }) \&\left(t<t^{\prime} \supset \boldsymbol{P}_{a_{i}}\left(F\left(t^{\prime}\right)\right) \geq \boldsymbol{P}_{a_{i}}(F(t))\right)\right.
\end{aligned}
$$

There is a production start point: $t^{P} \leq T$ :

$$
\forall t \leq t^{P}\left((F(t))=\boldsymbol{P}_{a_{i}}(\text { Begin })\right.
$$

If $t^{P}=T$, then the resource is not produced. 
2. Over the entire trajectory, the resource is consumed.

$$
\begin{aligned}
& \forall t\left(f_{i}(t) \leq 0\right) \\
& \forall t, t^{\prime}\left(\boldsymbol{P}_{a_{i}}(F(t)) \leq \boldsymbol{P}_{a_{i}}(\text { Begin }) \&\left(t<t^{\prime} \supset \boldsymbol{P}_{a_{i}}(F(t)) \leq \boldsymbol{P}_{a_{i}}\left(F\left(t^{\prime}\right)\right)\right)\right.
\end{aligned}
$$

There is a moment of start of consumption: $t^{C} \leq T$ :

$$
\forall t \leq t^{C}\left(\boldsymbol{P}_{a_{i}}(F(t))=\boldsymbol{P}_{a_{i}}(\text { Begin })\right.
$$

If $t^{C}=T$, then the resource is not consumed.

3. On the trajectory, the resource is first only consumed, and subsequently produced and possibly consumed.

In the notation of points 1, 2 and taking into account the validity of (1.2) and (2.2) at suitable time intervals from $[0, T]$, the situation is characterized by the following statements:

$$
\begin{aligned}
& \forall t \in\left[0, t^{P}\right]\left(f_{i}(t) \leq 0\right) \\
& \forall t \in\left[0, \min \left(t^{C}, t^{P}\right)\right]\left(f_{i}(t)=0\right)
\end{aligned}
$$

There is a moment $t^{C=P}$ of satisfying the demand for a resource due to own production: $t^{P} \leq t^{C=P} \leq T$ :

$$
\forall t \in\left[0, \max \left(t^{C}, t^{C=P}\right)\right]\left(f_{i}(t) \leq 0\right) \& \forall \mathrm{t} \in\left[\max \left(t^{C}, t^{C=P}\right), T\right]\left(f_{i}(t) \geq 0\right)
$$

Figure 3 shows examples of graphs of the functions $f_{i}(t)$, showing possible situations of projection of the trajectory associated with the factor $a_{i}$.

Projections $\boldsymbol{P}_{a_{i}}$ for each $i \in\{1, \ldots, n\}$ give an idea of how factors influence the formation of the development criterion $F$ separately. They make it possible to identify sections of the trajectory where the factor ai is significant (in the sense of definition, from Section 3). This is what makes it possible to clarify the phase structure of the process constructed on the basis of the relationship of materiality. Transitions of the trajectory through points separating the phases are a change in the groups of the most strongly influencing factors.
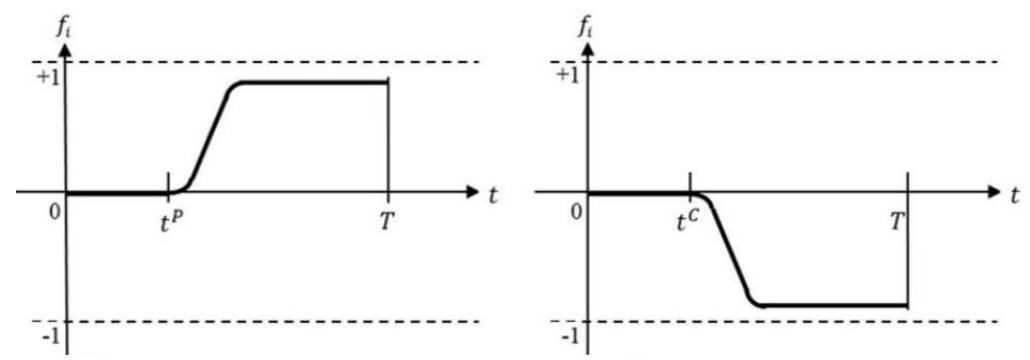

1) Resource begins to be produced. 2) Resource begins to be consumed.

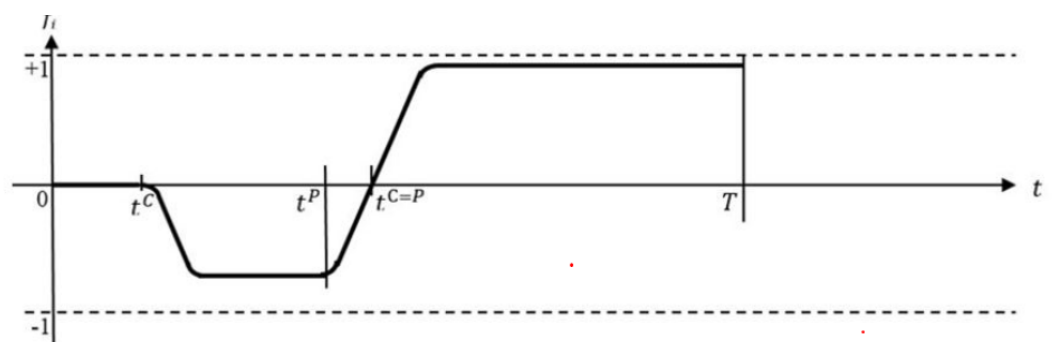

Figure 3. Examples of graphs of the function $f_{i}(t)$ in different patterns of resource $a_{i}$ consumption and production. 
Figure 4 gives a conditional example of the projection of the trajectory onto the plane $\left(F, C\left(a_{1}, \ldots, a_{n}\right)\right)$. The shaded rectangle shows the projection of the development axis $\boldsymbol{P}_{a_{i}}($ Begin, End $)$, $\alpha$ is its angle of inclination to $C\left(a_{1}, \ldots, a_{n}\right)$. The image of the admissibility region is a dotted rectangle, for clarity, somewhat shifted relative to the rectangle of the projection of the development axis. White points on the trajectory mark phase transitions (in the example, they are randomly placed along the curve). A thick cross shows the "plumage" of the arrow of time.

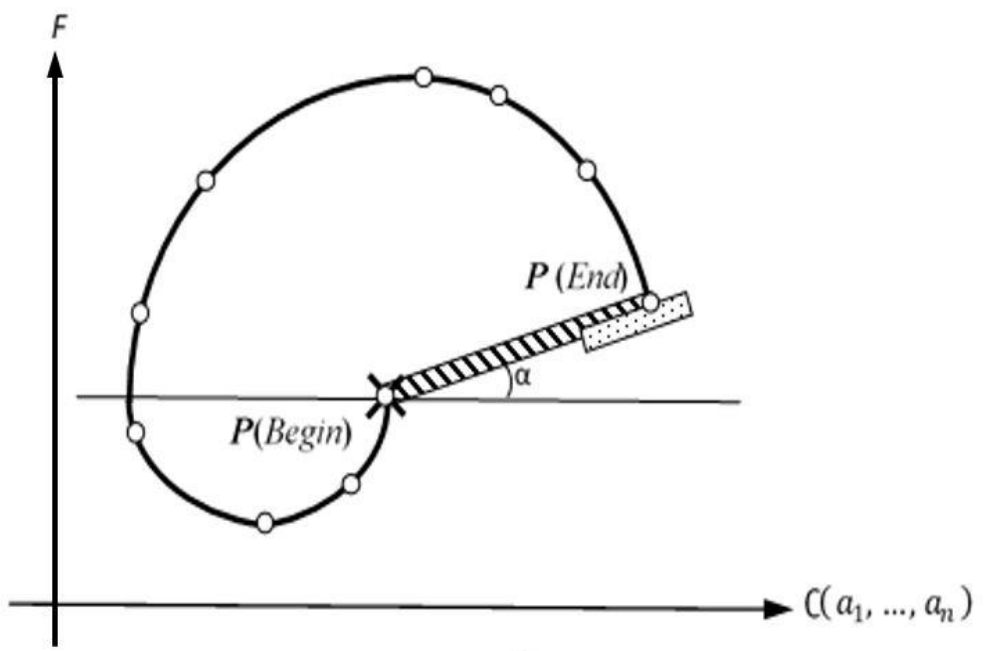

Figure 4. The projection of the trajectory onto the plane $\left(F, C\left(a_{1}, \ldots, a_{n}\right)\right)$.

\section{Time when modelling developing processes}

Projections of the trajectory on the plane $\left(F, C\left(a_{1}, \ldots, a_{n}\right)\right)$, i.e. spiral motion in the direction of the vector $\boldsymbol{P}($ Begin, End $)$, can be considered as a representation of the time of development of the process. Time here should be understood not as hours, days, months, etc., evenly distributed along a special axis, but as a sequence of events that occur during the development of a process. The occurrence of some events and their sequence can be predicted. It is how the phases of the trajectory appear: they are areas of the greatest influence of certain groups of factors, and phase transitions are the boundaries of these areas. Their identification becomes possible due to the analysis of projections onto the plane (time, criterion) and on the planes $\left(F, f_{i}(t) *\left(0, \ldots, 0, a_{i}, 0, \ldots, 0\right)\right), 1 \leq i \leq n$.

The sequence of phases does not fix and, in principle, cannot fix the duration of phases indicated as gaps between events. This is possible only for a very narrow class of deterministic processes that are completely determined by suitable equations and the initial state of the data.

The above does not mean a rejection of the numerical parameters of time. If there is a need for this, then time indicators can be set as explicit attributes of events, phases, and other objects of the model, but with one condition: these attributes should not violate the events partial order that built during model construction.

In the general case, the duration of phases can be talked about historically, considering and analyzing the experience of the development of processes. This experience can be transferred to the analysis of other processes, but only if their features and, very importantly, the features of their functioning environment are taken into account. Based on such a transfer, forecasting appears. And again, the condition for correctness is to maintain a partial order of events.

The concept of time is redundant in most models of development; its role is played by local ordering of events. At the level of local actions of processes, we may identify time with the sequence of events that the processes generate and to which they react. Events related to one phase build the local time of this phase, and since the phase transition is also a consequence of the reaction of the process to a specific event, it sets a starting point for the local time of the next phase. 
This mechanism works (and much more accurately than non-deterministic clocks) if the events are considered quite widely, i.e. as any changes in the state of the system for which at least one reaction is provided when the condition for its actuation is fulfilled. For example, the accumulation of the value of a factor in excess of a certain limit for it should be considered an event for the process if this leads to a change in its trajectory compared to that which would be without the reaching the given limit. This clearly illustrates the modelling of operation of the incandescent lamp from Section 3. The fact that some events involve reactions associated with the generation of new events suggests that some events occurred earlier than others, but there is no reason to judge the simultaneity or consecution of events that are not related with acts of creation. In particular, if several reactions to the same even are provided, then the order of their execution is considered to be uncertain. More briefly, this means the absence of global uniform time for all system of processes of the that generate events and respond to them. Its role is played by a partial order defined on a multitude of events and reactions.

Details on time modelling in event-driven systems one can found in [6].

A similar to our position was presented by G. Shchedrovitsky back in 1975 in [7] in connection with the modelling of developing systems as one of the base concept of thought activities study. He speaks of «changing single acts of thought activity» that remain and exist invariably and «as if outside of time», and time itself (we would say "global time") appears only «... in the cognitive activity that we carry out in relation to [research] object».

If the process allows external influence on its development, then the factors associated with such an influence can accelerate, slow down or stop the process. When the external environment is able to purposefully change these factors, we can talk about management and planning, i.e. about targeted impacts. This case demonstrates situations in which it is worth using a criterion for the development of a process with an extended quality assessment: additional tracking of trajectories may be required at bifurcation points in which the state of the system changes.

In connection with the remarks on the concept of time, the reliability of plans based on projections of trajectories is rather low even for processes of the same type (with any understanding of what is "the same type"). Their role is in another: they make it possible to qualitatively test development options and evaluate the choice of a management strategy. The optimization control possible on the deterministic sections of the development paths does not provide any reference points for phase transitions, and it is for their generation that modelling based on the development spiral is useful.

\section{Snail of development and elements of the methodology for its use}

Presented in figure 4, the flat image format of a spiral of development is not convenient enough for a control job. For this reason, it is advisable to transform it in such a way as, firstly, to increase the visibility, and secondly, to enable the development attributes to be displayed on the diagram, i.e. factors and groups of influence factors. It is this format, called the snail of innovation, is proposed in the work of Badulin [2] as applied to cyclical processes in the economy. Transformation of the projection of the development trajectory onto the plane in order to approximate its presentation to the format of the snail is possible, and it reduces to the following steps:

1. Rotate the graph by $(90-\alpha)^{\circ}$ counterclockwise. This will prepare the imposition of a spiral on the watch dial with the usual position of XII in a vertical position.

2. Stretch and contract the graph in various directions, bringing the spiral closer to a circular shape. In this case, the projection of the development axis $\boldsymbol{P}_{a_{i}}($ Begin, End) becomes a vertical segment of ordinate axis that restricts the approximation to the circle.

3. Build the quadrants of the plane and mark on their axes the phase transitions inherited from the $S$-curve. This will combine our model with the S-curve model.

4. Mark additional phase transitions on the graph. As a result, the phases of the project can be represented as sectors of a circle. 


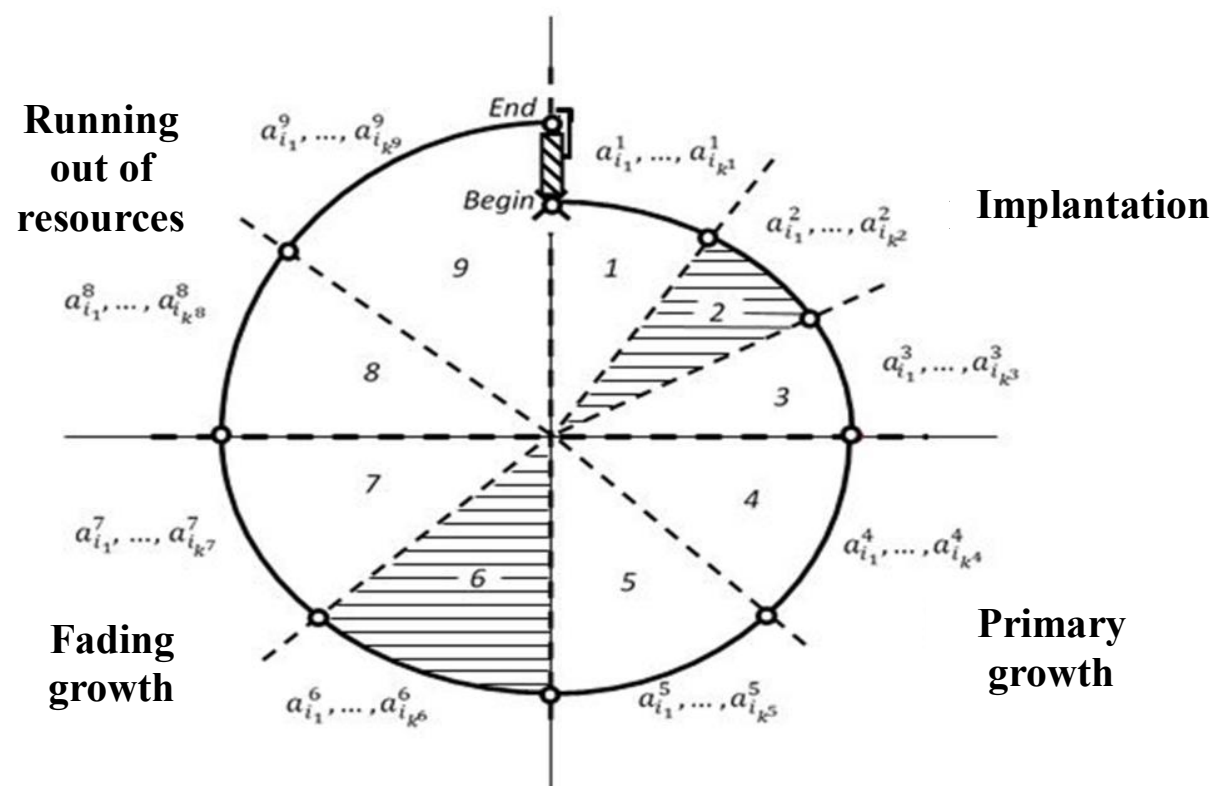

Figure 5. Snail of the process development.

This transformation deprives the image of the development criterion $\mathrm{F}$ of the time measure, but due to additional notation, it allows you to explicitly reflect the characteristics of each of the selected phases, which is very important for analysis. As an example in figure 5 shows the transformed representation of the projection of the development trajectory onto the plane $\left(F, C\left(a_{1}, \ldots, a_{n}\right)\right)$, shown in figure 4 . The sectors corresponding to the phases are numbered and separated from each other by dashed rays. The remaining notation of figure 4 are saved.

The development snail makes it possible to consider the phases of the process as autonomous processes - subprocesses of the integrating process and, in particular, to set the tasks of independent modelling of these components of the main process. At its core, this leads to a system of models that, by preserving relationships, ensures harmonization of calculations. The researcher has new means of analyzing the relationships between phases, due to geometric constructions: in addition to the obvious neighborhood relations, opposing sectors can be considered from the point of view of their mutual influence on each other. An example of the confrontation between sectors 2 and 6 highlighted in figure 4 by shading, can be interpreted as the influence of the results of the second phase on the quality of the sixth. It is clear that the task of oppositions is ambiguous, that for one sector it may be necessary to define several confrontations, and, finally, that the meaningful meaning of the oppositions does not necessarily exist or is not considered essential.

The experience of researching economic systems using the snail indicates that confrontations are useful for identifying actions in earlier sectors that are necessary for more efficient implementation of processes in opposing sectors. It is natural to expect a similar improvement in the quality of modelling by taking into account the confrontations and in the design of technical systems for which the behavior on stationary sections of the trajectory is carried out by methods of mathematical modelling with boundary conditions determined externally.

It is appropriate to note that the use of a snail as a representation of the development of processes is useful not only within the framework of a geometric metaphor. No less interesting in this respect is the more straightforward metaphor of the living snail, which is associated with consistent movement without inertia. Unlike a stone rolling from a mountain due to the energy stored at the top, each movement of the snail is a correction of its current state as a result of a reaction to the perceived state of 
the environment. If the snail decides to change state, a new direction of movement is initiated. Otherwise, the direction remains the same.

This behavior is similar to the management of well-established production, when it is enough to monitor changes in the situation and, based on the assessment of this, make decisions on whether the process needs correction, and if so, what actions should be taken. When deciding on a transition to a new state (there are not many such states in real snails), process control changes, but the essence of corrective control remains. This analogy points to the scope of adequate use of the snail as a tool to support the selection of process control options.

The metaphor of the development spiral as the basis for purposeful evolving processes modelling is significantly enriched by the geometric representation of the processes trajectories as curves in the cones of admissibility in the space of factors. Of course, this illustration should be considered only as an example of a framework that, for real use, needs to be supplemented with meaningful details: the properties of the modelling object, its representation as a system with its elements, etc.

\section{Conclusion}

The modelling of purposeful processes based on the geometric representation of their trajectories, presented in this work, uses quite understandable geometric constructions. At the same time, the given examples indicate rather good prospects for research in completely different applied fields. But for the practical application of the proposed approach, it is required to solve two interrelated research problems. Firstly, this is the creation of specialized tools to support the processing of information about the purposeful development of processes, represented by geometric abstractions, and secondly, it is the development of a methodology for using such tools.

The presented schemes that implement geometric modelling are given at the level of formalized abstraction without reference to the substantive aspects of modelling. This is done for the sake of clarity of demonstrating an approach, the development of which assumes an applied focus.

In [3], the requirements for tools for working with information about processes obtained using geometric constructions are formulated. It was shown how to define cones of trajectories, how to project trajectories onto a plane (time, criterion), how to extract information from these constructions. In the current article, the tools for working with forecasts and other aspects of studying reality have been significantly expanded, which allows us to offer a real methodology using them.

Remaining within the framework of a formalized abstraction, this methodology prescribes the organization of development modelling in the form of a sequence of the following actions:

- Determine the function of the development criterion of the simulated process, and the set of factors on which its implementation depends and which are available for measurement;

- Set points Begin and End that mark begin and end of the process monitoring, as well as the initial and target conditions for its implementation;

- Select the phases of the process and construct the factor spectrum of the criterion function, i.e. identify groups of factors which influence dominates in the phases of the process corresponding to the groups;

- Define projection of trajectories for the options for the process paths that are accepted as significant for the analysis and calculate with their help the influence of factors on the development of the process for different paths;

- Track phase opposing to identify the effects of earlier phases on subsequent development.

Of course, for a real application of the approach, it is required to indicate the types and classes of objects of the model system, as well as the means of their interaction. It is also necessary to define processes that implement aspect models producing and consuming data represented by common factors. This concretization should be provided for any simulation-based study. It includes the building of the architecture of the model, as well as the choice of modeling types for aspect models. This and many other things, that need to be borne in mind when conducting modelling purposeful processes, require 
own methods and tools, discussion which is beyond the scope of this work. Here we define the general typical structures of models of purposefully developing systems i.e. a kind of framework for this investigation work. The missing information on how the architecture of development models can be built, and what tools are required for this, can be found in [8].

In this work, we have not touched all on the issues related to the use of parallelism in modeling developing systems. The importance of this issue is understandable. However, we believe that there is no universal method for implementing parallel operating models. Instead, it is necessary to look for parallel solutions that are suitable for a specific research area. As an example of the implementation of this approach, it is pertinent to point to the publication by Il'in [9], which discusses various parallelism strategies in the field of mathematical modeling.

\section{Acknowledgments}

This work was carried out under state contract of the Ministry of Education and Science of the Russian Federation with The Institute of Computational Mathematics and Mathematical Geophysics SB RAS (0251-2021-0005)

\section{References}

[1] Badulin N 2012 "Innovation snail", "triple helix" and other circular processes in the economy". Innovatika-2012: Collection of materials of the VIII All-Russian school-conference of students, graduate students and young scientists with international participation (April 25 28, 2012). Vol 1 pp 7 - 29 (in Russian).

[2] Badulin N Economic theory of relativity or "innovation snail".

URL: Microsoft Word - 150615_Innovation_Snail-Article+ (triplehelixassociation.org).

[3] Skopin I 2015 The formalization of representations purposefully developing processes. System Informatics No 6 pp 35 - 52. URL: https://www.system-informatics.ru/en.

[4] S-Curve 2017 PMBOK ${ }^{\circledR}$ Guide and Standards - Sixth Edition. Project Management Institute: A guide to the project management body of knowledge. Newtown Square, PA. Online resource. ISBN 9781628253900

[5] Skopin I 2018 Phases and phase transitions in the modelling of purposefully clinical processes. Optimization problems for complex systems: Materials XIV international Asian Schoolseminar (20-31 July 2018). Part 2. Almaty, p. 208 - 217, (in Russian).

[6] Skopin I 2020 The concept of time when modelling evolving systems. Problems of Informatics No 4 pp 5-26. DOI: 10.24411 / 2073-0667-2020-10013 (in Russian).

[7] Shchedrovitsky G 1975 Design automation and tasks of design activities elaboration. Development and implementation of automated systems in designing (theory and methodology). Strojizdat pp. 3 - 77, (in Russian).

[8] Skopin I 2017 Tool Support of Developing Systems Simulation with Active Elements. Global Journal of Computer Science and Technology: A Hardware \& Computation.Vol 17 (USA) Online ISSN: 0975-4172 \& Print ISSN: 0975-4350 pp. $45-51$.

[9] Il' in V 2017 On the parallel strategies in mathematical modeling. Lecture notes, CCIS, Springer. Vol.753 pp.69-81 URL: https://icmmg.nsc.ru/sites/default/files/pubs/on the parallel strategies in mathematical modeling - 1 .pdf. 